\title{
Primer aislamiento de Mycobacterium bovis de búfalo del nordeste argentino
}

\author{
Guanziroli Stefani, M.C. ; Cicuta, M.E.; Zumárraga, M.J.'; Romano, M.I. ${ }^{2}$ \\ ${ }^{1}$ Cátedra de Microbiología, Facultad de Ciencias Veterinarias, UNNE, Sargento Cabral 2139, Corrientes (3400), \\ Argentina. Tel/Fax 03783-425753, E-mail: cicuta@vet.unne.edu.ar. \\ ${ }^{2}$ Instituto de Biotecnología, INTA Castelar (Argentina).
}

\begin{abstract}
Resumen
Guanziroli Stefani, M.C.; Cicuta, M.E.; Zumárraga, M.J.; Romano, M.I.: Primer aislamiento de Mycobacterium bovis de búfalo del nordeste argentino. Rev. vet. 19: 2, 143-146, 2008. Para detectar búfalos infectados con Mycobacterium bovis se realizaron pruebas tuberculínicas intradérmicas a 402 búfalos de raza Murrah del nordeste argentino. Cuatro animales resultaron positivos y seis sospechosos a la prueba ano-caudal simple, de los cuales cinco fueron positivos a la prueba cervical comparada. Se realizó necropsia a una hembra de 9 años que había reaccionado positivamente a ambas pruebas, en la que se observó sólo un ganglio retromamario afectado con necrosis caseosa. A partir del mismo se aisló M. bovis espoligotipo 34, que es el más frecuente en los aislamientos provenientes del ganado bovino de la zona. Se destaca la ubicación del linfonódulo afectado para tenerla en cuenta en las necropsias de la especie bubalina.
\end{abstract}

Palabras clave: búfalo, Mycobacterium bovis, tuberculosis bovina.

\begin{abstract}
Guanziroli Stefani, M.C.; Cicuta, M.E.; Zumárraga, M.J.; Romano, M.I.: First isolation of Mycobacterium bovis from buffalo of the North East of Argentina. Rev. vet. 19: 2, 143-146, 2008. In order to detect buffalos infected with Mycobacterium bovis, 402 animals of Murrah breed were tested by means of intradermal tuberculin. Four resulted positive and six suspicious to the caudal fold simple test; from these, five were positive to comparative cervical test. One nine years-old positive female was necropsied. The only granulomatous lesion found was in one retromammary lymph-node. From this, M. bovis spoligotype 34 was isolated. This genetic type is the predominant in cows of this region. We emphasize the localization of this lesion, which is not usually found in cattle.
\end{abstract}

Key words: buffalo, Mycobacterium bovis, bovine tuberculosis.

\section{INTRODUCCIÓN}

La tuberculosis bovina es una enfermedad infecciosa causada por Mycobacterium bovis, a la que el búfalo es también susceptible. En esta especie ha sido reportada en Italia, Brasil, India y Australia 8, 9, 13, 16, 22, 23, 27. Esta enfermedad crónica adquiere relevancia frente a la longevidad de los bubalinos, cuya vida productiva se puede extender a veinte años, proporcionando mayores posibilidades de desarrollo y transmisión de la misma ${ }^{11,24}$. Los signos clínicos pueden estar ausentes en los primeros estadios de la enfermedad que, siendo una zoonosis, se transforma en riesgo ocupacional para quienes trabajan con animales, como veterinarios, tamberos, trabajadores rurales, personal de frigoríficos y otros ${ }^{21,26,28}$.

Recibido: 6 octubre 2008 / Aceptado: 10 noviembre 2008
En el animal vivo el diagnóstico se efectúa por examen clínico y por medio de reacciones de hipersensibilidad retardada (prueba tuberculínica) efectuada con derivado proteico purificado (purified protein derivate, $P P D)$ obtenido de cepas aviares $(P P D-\mathrm{A})$ o bovinas $(P P D-\mathrm{B}){ }^{25}$. Los tests tuberculínicos presentan una sensibilidad entre 72 y $96 \%$ y una especificidad de $70-99 \%$ para los bovinos, estos índices no parecen ser válidos para los bubalinos dado que reaccionan en forma más exagerada a las micobacterias ${ }^{17,20,27}$. Se ha demostrado que la prueba caudal simple posee sensibilidad de $81,8 \%$ y especificidad de $93,3 \%$ en bovinos y que, con el aumento de la dosis a $0,4 \mathrm{mg}$, la sensibilidad alcanzó a la especificidad, afirmándose que, entre las pruebas simples, la cervical proporciona el máximo de sensibilidad y la caudal el máximo de especificidad ${ }^{7}$. Algunos animales pueden no responder a la tuberculina debido a diversos factores como infección reciente, fin de ges- 
tación o desnutrición, ocasionando resultados falsos negativos ${ }^{12}$. También en avanzado estado de infección, pueden manifestar el fenómeno de anergia, o ausencia de reactividad cutánea ${ }^{15}$. La respuesta de los bovinos comúnmente se inicia a los 30-50 días post-infección.

Debido a que las reacciones de los bubalinos muestran mayor intensidad que las de los bovinos, la lectura de la induración no debe seguir los mismos parámetros de los últimos ${ }^{13,14,18}$. Al referirse a la prueba comparativa, que utiliza simultáneamente $P P D$ aviar y bovino en el mismo animal, algunos autores sostienen su utilidad para diferenciar animales infectados por $M$. bovis de aquéllos expuestos a otras micobacterias o géneros afines ${ }^{9}$. En presencia de mayor reactividad a la tuberculina aviar (que representa sensibilidad inespecífica), las reacciones a la tuberculina bovina se consideran poco significativas en los bovinos ${ }^{2}$. La prueba es considerada positiva en esta especie cuando la respuesta al $P P D-B$ es $4 \mathrm{~mm}$ igual o mayor que el PPD-A (de 1 a $4 \mathrm{~mm}$ es sospechoso) ${ }^{26}$. La concentración recomendada para bovino es de 2.000 UI de $P P D-B$ (usualmente $0,1 \mathrm{mg} / \mathrm{ml}$ ) determinada por ensayo biológico frente a un patrón de referencia internacional ${ }^{19}$. El test tuberculínico no es efectivo en todas las especies; algunos investigadores afirman que no es un buen método de diagnóstico para utilizar en rodeos bubalinos debido a que en muchos animales reactores no se ha podido confirmar la infección a la necropsia ${ }^{17}$.

Se ha registrado sensibilidad del $75 \%$ en la prueba comparativa contra $87,5 \%$ en la simple cervical y una sensibilidad semejante en la caudal ${ }^{12}$. Aumentándose la dosis de tuberculina aplicada, la sensibilidad aumenta, manteniéndose la especificidad, aún así no se puede alcanzar el $100 \%$ de sensibilidad debido a la presencia de animales anérgicos en el rebaño. La sensibilidad y especificidad pueden ser alteradas modificándose la dosis inyectada, la concentración en unidades internacionales o los valores límites de respuesta o puntos de corte de reacción ${ }^{7,12,20}$.

Se recomienda la lectura por medición en la prueba ano-caudal en el búfalo, considerando reacción positiva cuando es mayor o igual a $10 \mathrm{~mm}$, sospechosa cuando sus valores se sitúan entre 6,4 y $9,9 \mathrm{~mm}$ y negativa si es menor a $6,3 \mathrm{~mm}^{23}$. También se sugiere que en la prueba cervical comparativa, la media aritmética de las reacciones específicas en bubalinos sea de 13,07 $\mathrm{mm}$ y en bovinos 7,81 mm. Así, la relación entre las medias de las dos especies es igual a 1,69. En bubalinos la reactividad cutánea en el pliegue ano-caudal fue 1,8 veces más intensa que la observada en la región cervical Los tests caudal simple y cervical comparativo en bubalinos naturalmente expuestos a $M$. bovis no fueron capaces de detectar todos los animales infectados y también fueron positivos en animales no comprobadamente infectados. En el trabajo citado se utilizó la técnica de reacción en cadena de la polimerasa (polimerase chain reaction o $P C R$ ) para la identificación de micobacterias del complejo $M$. tuberculosis aisladas de linfonódulos de bubalinos naturalmente infectados, visto que, debido al crecimiento muy pobre en medios de cultivo, no fue posible la confirmación de la especie de micobacteria a través de pruebas bioquímicas y de cultivo ${ }^{23}$.

Luego de la necropsia, el diagnóstico de tuberculosis se confirma a través de exámenes histopatológicos y bacteriológicos, a los que se suman las técnicas de biología molecular (sondeos de ADN y $P C R)^{9}$. La técnica de $P C R$ está siendo considerada como el avance más promisorio en el diagnóstico rápido de tuberculosis, capaz de reducir las 4 a 8 semanas necesarias para el desarrollo del cultivo, a 1 o 2 días para la obtención de resultados, siendo aplicada en la identificación de micobacterias directamente, a partir de muestras clínicas, con una sensibilidad y especificidad muy elevadas, ya que es capaz de detectar menos de 10 bacilos en la muestra ${ }^{1,4-6}$.

En base a estudios realizados en diversos países se considera necesario establecer parámetros específicos para los búfalos, tomándose en cuenta las diferencias de respuesta inmune entre bovinos y bubalinos. Algunos investigadores afirman que es posible extrapolar los resultados de investigaciones sobre diagnósticos de bovinos a bubalinos 11,13,17, 18, 21 , aún así, deben considerarse las posibles diferencias inmunológicas entre las especies, visto que muchas veces se toman valores de bovinos que no pueden necesariamente presentar la realidad, incurriendo en sacrificios innecesarios y pérdidas económicas.

Dado que es importante que el control de la tuberculosis sea aplicado a todas las especies susceptibles, el objetivo del presente trabajo fue tipificar la cepa de micobacteria aislada luego de la realización de las pruebas tuberculínicas en búfalos, acompañada del diagnóstico histopatológico y pruebas de biología molecular.

\section{MATERIAL Y MÉTODOS}

Se realizó la prueba tuberculínica intradérmica simple ano-caudal a 402 búfalos de raza Murrah de la provincia de Corrientes, con un rango de edad de 1 a 20 años, a los cuales previamente se les tomó la medida del grosor cutáneo del pliegue externo con calibre milimetrado. Se utilizó $0,1 \mathrm{ml}$ de $P P D-\mathrm{B}$ con una concentración proteica de $1 \mathrm{mg} / \mathrm{ml}(32.500 \mathrm{UI} / \mathrm{ml})$, provisto por el Servicio Nacional de Sanidad Animal (SENASA) ${ }^{26}$. Las lecturas se realizaron a las $72 \pm 6$ horas, interpretando la induración de la siguiente manera: positivos con diámetro igual o mayor de $10 \mathrm{~mm}$, sospechosos entre $6 \mathrm{y}$ 9,9 mm y negativos iguales o menores a $5,9 \mathrm{~mm}^{23}$.

A los animales positivos y sospechosos se les repitió la prueba cervical comparada en un plazo de 60 días, interpretándose como positivos a aquéllos que registraban una diferencia de $P P D-\mathrm{B}$ con respecto a $P P D-\mathrm{A}$ igual o mayor de $5 \mathrm{~mm}$, sospechosos entre 3 y 4,9 $\mathrm{mm}$ y negativos debajo de $3 \mathrm{~mm}^{23}$.

Se realizó una necropsia examinando histopatológica y bacteriológicamente los linfonódulos y órganos afectados. La metodología utilizada fue la habitual, realizándose tinciones con hematoxilina-eosina, Grocott, PAS y Ziehl-Neelsen para observación de bacilos 
ácido-alcohol resistentes (BAAR), así como procesamiento bacteriológico para aislamiento de micobacterias: decontaminación de la muestra utilizando el método de Petroff con hidróxido de sodio al $4 \%$, centrifugación, neutralización y siembra en los medios de Stonebrink y Löwenstein-Jensen.

Una ansada del aislamiento fue resuspendida en agua e incubada a $95^{\circ} \mathrm{C}$ durante 45 minutos; $5 \mu 1$ del sobrenadante fueron utilizados para la amplificación de la región $D R$ por $P C R$ para la tipificación por la técnica de spoligotyping, según protocolo ${ }^{10}$.

\section{RESULTADOS Y DISCUSIÓN}

De los 402 animales tuberculinizados, 4 (0,99\%) fueron positivos a la prueba ano caudal simple con reacciones de 11 a $17 \mathrm{~mm}$ y $6(1,49 \%)$ resultaron sospechosos, con rangos de 6 a $8 \mathrm{~mm}$, totalizando 10 reaccionantes $(2,48 \%)$. De los 10 animales positivos y sospechosos a la prueba ano caudal simple a quienes se les efectuó la cervical comparada, 5 resultaron reaccionantes con predominancia de respuesta al $P P D-\mathrm{B}$ con rangos de diferencias entre 7 y $31 \mathrm{~mm}$ con respecto al $P P D-\mathrm{A}, 2$ sospechosos y 3 negativos.

Se realizó necropsia a una hembra de 9 años que había reaccionado positivamente a las pruebas ano caudal (17 mm) y cervical comparada (16 mm de diferencia entre $P P D-\mathrm{B}$ y $P P D-\mathrm{A})$. En la misma se observó sólo un ganglio retromamario afectado con necrosis caseosa; el resto de los órganos carecía de lesiones compatibles con tuberculosis. El diagnóstico histopatológico del ganglio retromamario (Figura 1) fue compatible con tuberculosis; el resto (retrofaríngeos, preescapulares, submaxilares, mesentéricos y poplíteos) presentó hiperplasia linfoide difusa. Se obtuvo aislamiento de $M$. bovis (Figura 2) sólo a partir del ganglio retromamario. Por spoligotyping se detectó el espoligotipo 34 (Figura 3), que abarca al $54,7 \%$ de $M$. bovis sobre un total de 219 aislamientos estudiados provenientes de ganado bovino de la zona ${ }^{3}$.

La tuberculinización en el búfalo presenta algunos inconvenientes como inexistencia del pliegue ano-caudal interno y heterogeneidad en la morfología del pliegue ano-caudal externo, en el cual el tamaño y grosor de la piel son muy variables. Presenta mayor cantidad de tejido laxo con diferente conformación histológica debido al mayor número de fibras elásticas por lo que es esperable que haya mayor reacción cutánea. El bubón intradérmico que se forma puede observarse fácilmente o no, dependiendo si el animal presenta la piel más gruesa o más fina en la zona, por eso es de suma importancia la utilización de calibre milimetrado.

Nuestras mediciones de induraciones concuerdan con las obtenidas en trabajos realizados con anterioridad $13,17,18,20,24,27$, donde se comprueba que la reacción es más pronunciada en la especie bubalina que en la bovina, por lo que los parámetros de medición de las pruebas también deben considerarse diferentes a los del bovino. Los establecidos por investigadores brasileros parecen ser acertados aunque nos faltaría corroborarlos con mayor número de necropsias en búfalos ${ }^{23}$.

El hallazgo de $M$. bovis con espoligotipo correspondiente al predominante en bovinos indica la diseminación clonal de esta cepa a la especie bubalina. Esto tiene un gran significado epidemiológico ya que está evidenciando el contagio entre bovinos y bubalinos, a diferencia de lo que ocurre en Brasil donde las cepas de $M$. bovis aisladas de búfalos tienen sus espoligotipos exclusivos, diferentes a los del bovino ${ }^{29}$. Ello debe alertarnos respecto a que la fuente de infección para el búfalo de esta zona serían los bovinos infectados. Es dable destacar que los campos lindantes a los habitados por búfalos se encuentran poblados de bovinos que cir-

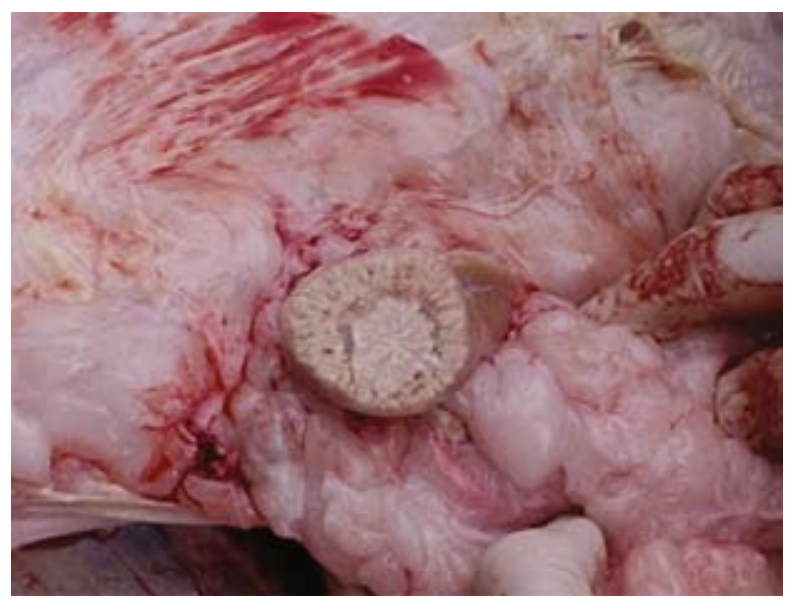

Figura 1. Ganglio caseoso retromamario del cual se obtuvo desarrollo de M. bovis.

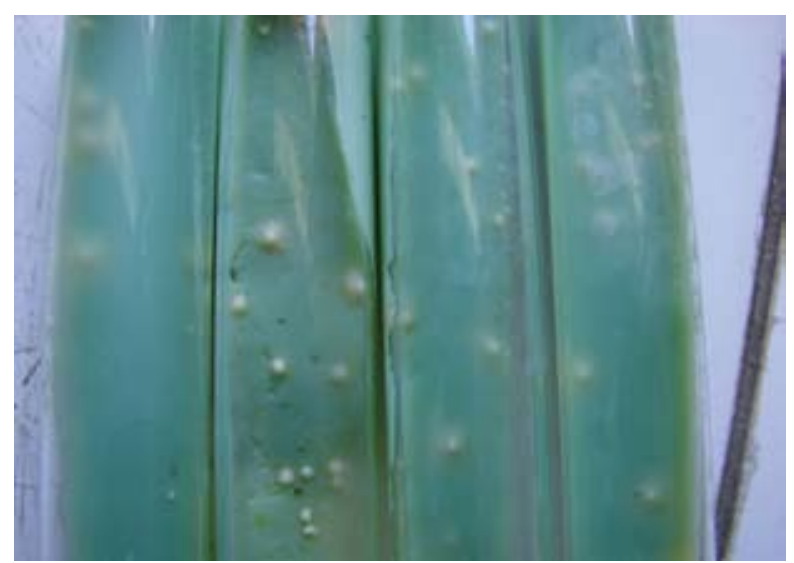

Figura 2. Cuatro tubos de medio de Stonebrink con colonias de $M$. bovis

\section{IIIIIIIIIIIIIIIIIII}

12345678910111213141516171819202122232425262728293031323334353637383940414243

Figura 3. Espoligotipo del aislamiento de Mycobacterium bovis procedente de búfalo. Los espaciadores se indican con números. 
cunstancialmente invaden los establecimientos.

La permanencia de esta enfermedad limita las posibilidades del sector pecuario y la comercialización internacional, influyendo negativamente en la rentabilidad de las explotaciones, en la calidad de los productos y en el consumo y la salud humana, de allí la importancia que el Plan Nacional de Control y Erradicación de la Tuberculosis Bovina, implementado por el SENASA en 1994, sea llevado a cabo y se implementen los parámetros más adecuados para ser aplicados a búfalos debido al incremento de la explotación de esta especie, muy valorada por la resistencia innata al calor de la región, precocidad, calidad de su carne y derivados lácteos.

\section{REFERENCIAS}

1. Beige J, Lokies J, Schaberg T, Finckh U, Fischer M, Mauch H, Lode H, Kohler B, Rolfs A. 1995. Clinical evaluation of a Mycobacterium tuberculosis PCR assay. $J$ Clin Microbiol 33: 90-95.

2. Centro Panamericano de Zoonosis. 1989. Tuberculosis: guía para proyectos de tuberculosis bovina. Organización Panamericana de la Salud, Nota Técnica $N^{\circ} 15$.

3. Cicuta ME. 2002. Epidemiología molecular de Mycobacterium bovis en la región nordeste argentina. Tesis doctoral, Universidad Nacional del Nordeste, Corrientes (Argentina), $58 \mathrm{p}$.

4. Claridge JE, Shawar RM, Shinnick TM, Plikaytis BB. 1993. Largescale use of polimerase chain reaction for detection of Mycobacterium tuberculosis in a routine mycobacteriology laboratory. J Clin Microbiol 31: 2049-2056.

5. Diaz R, Monitoro E, Maestre JL, Echemendia M, Valdivia JA. 1994. Polimerase chain reaction for the detection of Mycobacterium tuberculosis complex. Mem Inst Osw Cruz 89: 211-212.

6. Forbes BA, Hicks KE. 1993. Direct detection of $M y$ cobacterium tuberculosis in respiratory specimens in a clinical laboratory by polymerase chain reaction. $J$ Clin Microbiol 31: 1688-1694.

7. Francis J, Seiler RJ, Wilkie IW, O'Boyle D, Lumsden MJ, Frost AJ. 1978. The sensitivity and specificity of various tuberculin test using bovine PPD and other tuberculins. Vet Rec 4: 4205.

8. Freitas J, Guerra JL, Panetta JC. 2001. Characteristics of the tuberculosis in slaughtered water buffaloes. Braz J Vet Res Anim Sci 38: 170-176.

9. Haagsma J. 2000. Bovine tuberculosis. In: Manual of Standards for Diagnostic Tests and Vaccines (Ed. Office International des Epizooties), Paris, p. 359-370.

10. Kamerbeek J, Schouls L, Kolk A, van Agterveld M, van Soolingen D, Kuijper S, Bunschoten A, Molhuizen H, Shaw R, Goyal M, van Embden J. 1997. Simultaneous detection and strain differentiation of Mycobacterium tuberculosis for diagnosis and epidemiology. J Clin Microbiol 35: 907-914.

11. Kanameda M, Ekgatat S, Wongkasemjit SC, Achimasiri TP, Kongkrong C, Buchaphan K, Boontarat B. 1999. An evaluation of tuberculin skin tests used to diagnose tuberculosis in swamp buffaloes. Prev Vet Med 39: 129-135.
12. Kantor I, Odeon AC, Stefani PE, Aza MJ, Madrid CR, Marchevsky N. 1984. Sensitivity of the cervical and the caudal fold tuberculin tests with Mycobacterium bovis in infected cattle of Argentina. Rev Sci Tech 3: 137-150.

13. Lau HD. 1994. Important economic diseases in buffaloes. Proceedings IVth World Buffalo Congreso, Sao Paulo (Brasil), p. 209-220.

14. Mohan RN. 1968. Diseases and parasites on buffaloes. Vet Bull 38: 647-659.

15. Monaghan ML, Doherty ML, Collins JD, Kazda JF, Quinn PJ. 1994. The tuberculin test. Vet Microbiol 40: 111-124.

16. Mota PM, Lobato FC, Assis RA, Lage AP, Parreiras PM, Leite RC. 2002. Ocurrencia de tuberculosis en rebaños bubalinos en Municipio de Parintis, Amazonas. Arq Bras Med Vet Zootec 54: 4.

17. Nain SP, Kaushik RK. 1985. Examination of tuberculinpositive animals. Indian J Anim Sci 55: 877-878.

18. Nicoletti P. 1994. An update on selected diseases which affect buffaloes. Proceedings IVth World Buffalo Congress, Sao Paulo (Brasil), p. 221-228.

19. Office International des Epizooties (OIE). 1992. Manual of standards for diagnostic tests and vaccines: bovine tuberculosis, Publ. OIE, Paris, p. 287-295.

20. Organización Panamericana de la Salud (OPS/OMS). 1992. Plan de acción para la erradicación de la tuberculosis bovina en las Américas. OPS/OMS, HPV/TB No 113, 41 p.

21. O'Reilly LM, Daborn CJ. 1995. The epidemiology of Mycobacterium bovis infections in animals and man: a review. Tuberc Lung Dis 76: 1-46.

22. Portugal MA, Giorgi W, Siqueira PA. 1971. Ocurrencia da tuberculose em bubalinos no Estado de Sao Paulo. Arch Inst Biol (Sao Paulo) 4: 231-238.

23. Roxo E. 1997. Tuberculose e brucelose em búfalos. Publ. Instituto de Investigación Sao Paulo (Brasil), p. 185-192.

24. Roxo E, Amaral X. 1998. Surto de tuberculose em bufalos no Estado de Sao Paulo. Publ. Instituto Biológico, Laboratorio de Tuberculose 65: 92.

25. Servicio Nacional de Sanidad Animal (SENASA). 1994. Plan nacional de control y erradicación de la tuberculosis bovina. Resolución $\mathrm{N}^{\circ}$ 1287. Publ. SENASA, Buenos Aires, Argentina, $78 \mathrm{p}$.

26. Servicio Nacional de Sanidad Animal (SENASA). 2000. Tuberculosis bovina, pruebas tuberculínicas (inoculación, lectura e interpretación), preguntas y respuestas. Publ. SENASA, Buenos Aires, Argentina, $76 \mathrm{p}$.

27. Vanamaya P, Sharma AK, Parai TP, Paliwal OP, Parihar NS. 1987. Evaluation of tuberculin and johnin tests with pathological lesions in buffaloes. Indian J Anim Sci 3: 189-190.

28. World Health Organization. 1993. Zoonotic tuberculosis. Report of the WHO meeting on zoonotic tuberculosis ( $M y$ cobacterium bovis), WHO/CDS/VPH Monograph Series 130: 27.

29. Zumárraga M, Martín C, Samper S, Alito A, Latini O, Bigi F, Roxo E, Cicuta ME, Errico F, Castro Ramos M, Cataldi A, Van Soolingen D, Romano MI. 1999. Usefulness of spoligotyping in the molecular epidemiology of $M y$ cobacterium bovis-related infections in South American countries. J Clin Microbiol 60: 251-257. 